\title{
New Stability Tests for Discretized Fractional-Order Systems Using the Al-Alaoui and Tustin Operators
}

\author{
Rafał Stanisławski $(\mathbb{D}$, Marek Rydel, and Krzysztof J. Latawiec $\mathbb{D}$ \\ Department of Electrical, Control and Computer Engineering, Opole University of Technology, ul. Prószkowska 76, \\ 45-758 Opole, Poland \\ Correspondence should be addressed to Rafał Stanisławski; r.stanislawski@po.opole.pl
}

Received 17 May 2018; Accepted 18 September 2018; Published 7 November 2018

Academic Editor: Rongqing Zhang

Copyright (C) 2018 Rafał Stanisławski et al. This is an open access article distributed under the Creative Commons Attribution License, which permits unrestricted use, distribution, and reproduction in any medium, provided the original work is properly cited.

\begin{abstract}
This paper provides new results on a stable discretization of commensurate fractional-order continuous-time LTI systems using the Al-Alaoui and Tustin discretization methods. New, graphical, and analytical stability/instability conditions are given for discretetime systems obtained by means of the Al-Alaoui discretization scheme. On this basis, an analytically driven stability condition for discrete-time systems using the Tustin-based approach is presented. Finally, the stability of discrete-time systems obtained by finite-length approximation of the Al-Alaoui and Tustin operators are discussed. Simulation experiments confirm the effectiveness of the introduced stability tests.
\end{abstract}

\section{Introduction}

Stable discretization of continuous-time fractional-order systems is an important issue in various areas of science and technology, including system science, signal processing, and control theory. In this field, we have three main discretization operators which can generate discrete-time counterparts for continuous-time fractional-order systems, in terms of the Euler, Tustin, and Al-Alaoui methods. There are two main problems to be solved during the discretization process for fractional-order systems. Firstly, the three discretization schemes lead to infinite complexity of rational, discrete-time counterparts of fractional-order derivative. Therefore, in practical applications various finite-length approximations of the discretization operators have been used, involving the most popular finite fractional difference (FFD) approximation in the Euler approach $[1,2]$ and finite-length implementations of the continuous fraction expansion (CFE) method in the Tustin and Al-Alaoui approaches [2-6]. Also, a number of papers have presented some other approximation/discretization methods for the fractional-order derivative [7-10].
Secondly, it is well known that the discretization process affects stability conditions for the discrete-time counterparts of continuous-time fractional-order systems [11-14]. Moreover, the stability conditions of fractional-order discretetime systems depend on a type of operator used in the discretization process. This can be easily seen when we compare stability results for discrete-time systems obtained for the "forward-shifted" Euler operator $[15,16]$ with those for the classical backward Euler operator [13]. The first results in the area of stability analysis for discrete-time fractionalorder systems have been developed in [11], and they concern sufficient conditions only. More complete results have been obtained in a special case of discrete-time fractional-order positive systems [17-20]. Simple, analytical, necessary, and sufficient stability results for discrete-time systems have been obtained for both "forward-shifted" and backward Euler operators $[13,15,16]$. Specific, numerical stability results for discrete-time fractional-order systems based on the Euler expansion have been presented in [21-23]. On the other hand, it is well known that finite-length implementations of the Euler and Tustin operators affect the stability conditions for the underlying discrete-time systems $[2,11,13,15]$. 
In this paper, we introduce simple, either analytically driven or purely analytical stability tests for discrete-time systems obtained by the use of the Al-Alaoui operator. These results are then used to propose a stability test for systems based on the discretized Tustin operator, which can be considered as a special case of the Al-Alaoui approach. Also, practically oriented results for discretization using a finite-length implementation of the Tustin approach of [2] are extended to finite-length approximation using the Al-Alaoui method [24].

This paper is organized as follows. Having introduced in Section 1 the stability problem for discretized commensurate fractional-order continuous-time systems, the system representations based on the Euler, Tustin, and Al-Alaoui discretizers are given in Section 2. Section 3 presents new stability results involving both graphical and analytical criteria, which are the main results of the paper. Moreover, Section 3 adopts the analytical stability criterion for the Al-Alaoui operator to the Tustin-based one. Discussion on the stability of discretization based on finite-length approximations of the Al-Alaoui operator is presented in Section 4. Simulation examples of Section 5 confirm the effectiveness of the proposed stability results. Section 5 summarizes the achievements of the paper.

\section{Preliminaries}

Consider a linear continuous-time state space system of commensurate fractional order $\alpha \in(0,2)$ described by

$$
\begin{aligned}
{ }_{0} D^{\alpha} x(t) & =A_{f} x(t)+B u(t), \quad x_{0}=x(0), \\
y(t) & =C x(t),
\end{aligned}
$$

where ${ }_{0} D^{\alpha}$ denotes a fractional-order derivative of order $\alpha$; $x(t) \in \mathbb{R}^{n}, u(t) \in \mathbb{R}^{n_{u}}, y(t) \in \mathbb{R}^{n_{y}}$ are the state, control, and output vectors, respectively; and $A_{f} \in \mathbb{R}^{n \times n}, B \in \mathbb{R}^{n \times n_{u}}$, and $C \in \mathbb{R}^{n_{y} \times n}$ are the system matrices, with $n_{u}$ and $n_{y}$ being the number of inputs and outputs, respectively. Here, the fractional-order derivative will be described by three various representations, involving the Caputo, Riemann-Liouville, or Grünwald-Letnikov definitions. But regardless of the specific definition of the fractional-order derivative, assuming the zero initial conditions in (1), that is ${ }_{0} D^{k} x(0)=0$, for any $k \in \mathbb{R}$, the Laplace transform of system (1) is as follows:

$$
\begin{aligned}
s^{\alpha} X(s) & =A_{f} X(s)+\mathrm{BU}(s), \\
Y(s) & =C X(s) .
\end{aligned}
$$

In the specific SISO case, when $n_{u}=n_{y}=1$, the system of (2) can be described by the transfer function

$$
\begin{aligned}
G\left(s^{\alpha}\right)= & \frac{B\left(s^{\alpha}\right)}{A\left(s^{\alpha}\right)}=\frac{b_{m}\left(s^{\alpha}\right)^{m}+b_{m-1}\left(s^{\alpha}\right)^{m-1}+\cdots+b_{0}}{a_{n}\left(s^{\alpha}\right)^{n}+a_{n-1}\left(s^{\alpha}\right)^{n-1}+\cdots+a_{0}} \\
= & \frac{b_{m}\left(s^{\alpha}-\gamma_{1}^{f}\right)\left(s^{\alpha}-\gamma_{2}^{f}\right) \ldots\left(s^{\alpha}-\gamma_{m}^{f}\right)}{a_{n}\left(s^{\alpha}-\lambda_{1}^{f}\right)\left(s^{\alpha}-\lambda_{2}^{f}\right) \ldots\left(s^{\alpha}-\lambda_{n}^{f}\right)},
\end{aligned}
$$

where $A\left(s^{\alpha}\right)$ and $B\left(s^{\alpha}\right)$ are the coprime polynomials in the variable $s^{\alpha}$ and $\lambda_{j}^{f}, j=1, \ldots, n$, and $\gamma_{j}^{f}, j=1, \ldots, m$, are the poles and zeros of $G\left(s^{\alpha}\right)$, called $f$-poles and $f$-zeros, respectively (compare [16]). Note that the $f$-poles $\lambda_{j}^{f}, j=1$, $\ldots, n$, are the eigenvalues of the state matrix $A_{f}$.

In the discretization process, we seek for a discrete-time equivalent of the fractional-order system (2), in form of the $\mathscr{Z}$-transform

$$
\begin{aligned}
w(z) X(z) & =A_{f} X(z)+\mathrm{BU}(z), \\
Y(z) & =C X(z),
\end{aligned}
$$

where $w(z)$ will be used as a discrete-time model of $s^{\alpha}$. Alternatively, for the SISO case, we can obtain a discrete-time fractional-order transfer function in form of

$$
\begin{aligned}
G(w(z)) & =\frac{B(w(z))}{A(w(z))}=\frac{b_{m}(w(z))^{m}+b_{m-1}(w(z))^{m-1}+\cdots+b_{0}}{a_{n}(w(z))^{n}+a_{n-1}(w(z))^{n-1}+\cdots+a_{0}} \\
& =\frac{b_{m}\left(w(z)-\gamma_{1}^{f}\right)\left(w(z)-\gamma_{2}^{f}\right) \ldots\left(w(z)-\gamma_{m}^{f}\right)}{a_{n}\left(w(z)-\lambda_{1}^{f}\right)\left(w(z)-\lambda_{2}^{f}\right) \ldots\left(w(z)-\lambda_{n}^{f}\right)},
\end{aligned}
$$

with $\lambda_{\mathrm{j}}^{f}, j=1, \ldots, n$, and $\gamma_{j}^{f}, j=1, \ldots, m$, being the $f$-poles and $f$-zeros, respectively, of the system defined in (3). Three main discretizers are used here as discretization functions $w(z)$, namely, the Euler, Tustin, and Al-Alaoui operators. The Euler operator is used in two versions, that is, the backward Euler operator

$$
s^{\alpha} \approx w_{\mathrm{Eul}}(z)=\frac{1}{h^{\alpha}}\left(1-z^{-1}\right)^{\alpha},
$$

or the "forward-shifted" Euler one

$$
s^{\alpha} \approx w_{\mathrm{Eu} 2}(z)=\frac{1}{h^{\alpha}} z\left(1-z^{-1}\right)^{\alpha},
$$

with $h$ being the sampling period. The Tustin operator has the form

$$
s^{\alpha} \approx w_{\text {Tus }}(z)=\left(\frac{2}{h} \frac{1-z^{-1}}{1+z^{-1}}\right)^{\alpha}
$$

The Al-Alaoui operator $[25,26]$ is obtained through an assumption that the integration rule is realized as a weighted sum of the Tustin and backward Euler rules. This leads to the Al-Alaoui operator $[25,26]$

$$
s^{\alpha} \approx w(z)=\left(\frac{1+a}{h} \frac{1-z^{-1}}{1+a z^{-1}}\right)^{\alpha},
$$

where $a \in[0,1]$ is the weighting coefficient.

It has been presented in several papers that selection of the discretization operator in the discretization process 
affects the stability conditions for discrete-time systems $[11,13,16]$. For instance, the two Euler methods represented by (6) and (7) lead to quite different stability results for the discrete-time systems. A detailed stability analysis for discrete-time systems obtained by the use of the two Euler operators has been presented in $[13,15,16]$.

In this paper, a new stability analysis for systems obtained by the discretization process using the Al-Alaoui operator will be presented.

\section{Main Results}

Firstly, we introduce a graphical stability approach, based on the Al-Alaoui operator.

Theorem 1. The discrete-time fractional-order system (4) or (5), with $w(z)$ as in (9), is asymptotically stable if and only if all $f$-poles $\lambda_{j}^{f}$ of the system, $j=1, \ldots, n$, are outside the instability area

$$
\mathcal{S}=\left\{r\left(\frac{1+a}{(1-a) h}\left(1-e^{-i \varphi}\right)\right)^{\alpha}, \quad r \in[0,1], \varphi \in(0,2 \pi]\right\}
$$

where $\alpha \in(0,2)$ is the fractional-order of system (4) or (5), $a \in[0,1)$ is the weighting coefficient, and $\varphi$ is the argument of the function $w\left(e^{i \varphi}\right)=\left.w(z)\right|_{z=e^{i \varphi}}$.

Proof. Consider the discrete-time fractional-order system (4) or (5) with $w(z)$ as in (9). Taking into account that the $\mathrm{Al}$-Alaoui operator of (9) is meromorphic, the stability area with respect to $f$-poles depends on the contour of function $\left.w(z)\right|_{z=e^{i \psi}}, \psi=(0,2 \pi][13,15]$. Therefore, the bound contour of the stability/instability regions is as follows:

$$
\begin{aligned}
w\left(e^{i \psi}\right) & =\left(\frac{1+a}{h} \frac{1-e^{-i \psi}}{1+a e^{-i \psi}}\right)^{\alpha} \\
& =\left(\frac{1+a}{h}(\rho(\psi)+i \zeta(\psi))\right)^{\alpha},
\end{aligned}
$$

where $\rho(\psi)=(1-a)(1-\cos \psi) /\left(1+a^{2}+2 a \cos \psi\right), \zeta(\psi)=$ $((1+a) \sin \psi) /\left(1+a^{2}+2 a \cos \psi\right)$, and $\psi \in(0,2 \pi]$. Now, substituting $\rho(\psi)$ and $\zeta(\psi)$ of (12) into the circle equation with the center in the point $(1 /(1-a), 0)$, we obtain

$$
\begin{aligned}
\left(\rho(\phi)-\frac{1}{1-a}\right)^{2}+\zeta^{2}(\phi)= & \left(\frac{(1-a)(1-\cos \phi)}{1+a^{2}+2 a \cos \phi}-\frac{1}{1-a}\right)^{2} \\
& +\left(\frac{(1+a) \sin \phi}{1+a^{2}+2 a \cos \phi}\right)^{2} \\
= & \frac{1}{(1-a)^{2}} .
\end{aligned}
$$

So, for $a \in[0,1)$, the inner contour $\rho(\phi)+i \zeta(\phi)$ fulfills the circle equation with the center in $(1 /(1-a), 0)$ and radius

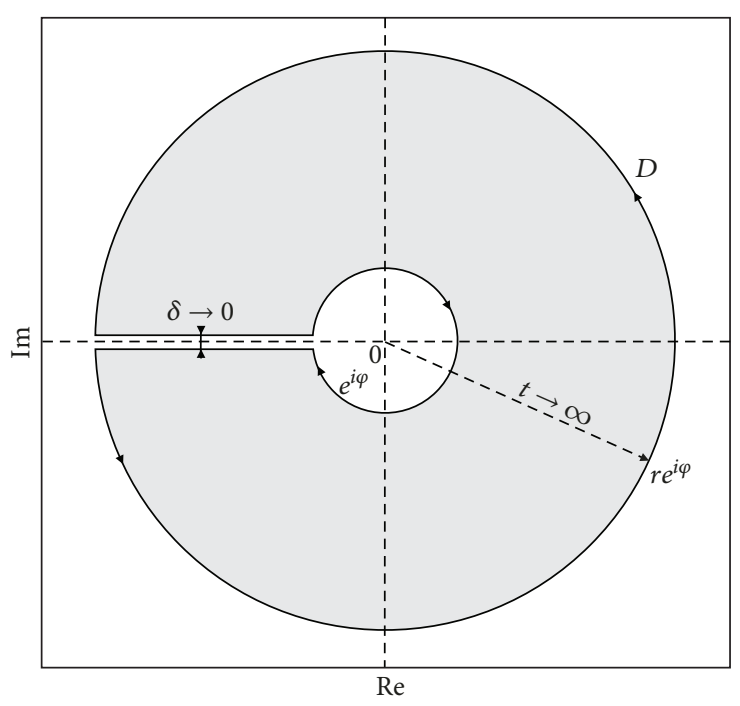

FIGURE 1: Simple closed positively oriented contour $D$.

$r=1 /(1-a)$. Therefore, we can write the same inner contour as a function of another parameter $\varphi$, that is,

$$
\rho(\varphi)+i \zeta(\varphi)=\frac{1}{1-a}\left(1-e^{i \varphi}\right), \quad \varphi \in(0,2 \pi]
$$

and finally we can describe the stability/instability contour for the Al-Alaoui operator of (12) as a function of $\varphi$ as

$$
w\left(e^{i \varphi}\right)=\left(\frac{1+a}{(1-a) h}\left(1-e^{-i \varphi}\right)\right)^{\alpha}, \quad \varphi \in(0,2 \pi] .
$$

The next steps of the proof follow the lines of Theorem 1 of [13]. Note that the characteristic equation of system (4) or (5)

$$
\left(w(z)-\lambda_{1}^{f}\right)\left(w(z)-\lambda_{2}^{f}\right) \ldots\left(w(z)-\lambda_{n}^{f}\right)=0
$$

is asymptotically stable if and only if all elements $w(z)-$ $\lambda_{j}^{f}, j=1, \ldots, n$, do not generate unstable poles in the $z$ domain. Accounting that $w(z)$ is a meromorphic function $(w(z)$ is homomorphic in $\mathscr{C} \backslash\{0\})$ and the contour $w\left(e^{i \varphi}\right)$ presented in (16) is a simply closed contour in the complex plane, we can prove the stability of the elements $w(z)-\lambda_{j}^{f}, j=1, \ldots, n$, on the basis on Cauchy's argument principle and Rouché's theorem [27].

Now, consider the simple closed contour $D$ as in Figure 1 whose interior domain is $\underline{C}=\mathscr{C} \backslash\left\{\mathrm{re}^{i \varphi}, 0 \leq r<1,0 \leq \varphi \leq 2 \pi\right\}$ such that $w(D) \neq 0$ and $w(D)<\infty$. Then, the winding number $W\left(w(D), \lambda_{j}^{f}\right)$ of $w(D)$ about $\lambda_{j}^{f}, j=1, \ldots, n$, that counts the number of times the curve $w(D)$ winds around the point $\lambda_{j}^{f}$ is

$$
W\left(w(D), \lambda_{j}^{f}\right)=\frac{1}{2 \pi i} \oint_{D} \frac{w^{\prime}(z)}{w(z)-\lambda_{j}^{f}} d z=n_{\mathrm{z}}-n_{\mathrm{p}}
$$




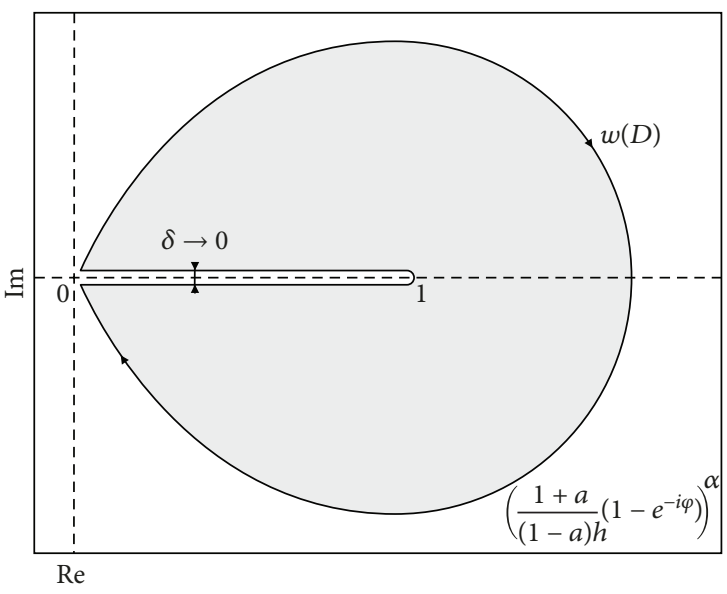

Figure 2: Contour of $w(D)$.

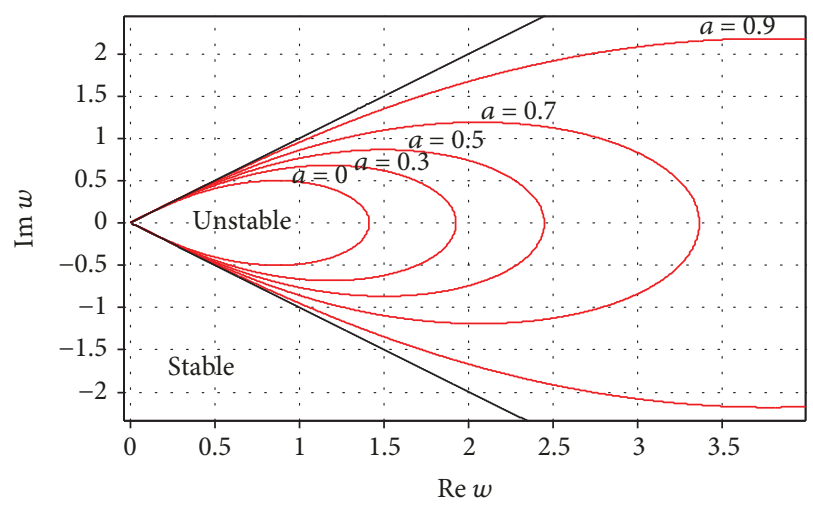

FIGURE 3: Stability/instability areas for $h=1$ and various $a$.

where $w^{\prime}(z)=(d w(z)) / d z$ and $n_{\mathrm{z}}$ and $n_{\mathrm{p}}$ denote the numbers of zeros and poles of $w(z)-\lambda_{j}^{f}$ inside the contour $D$, respectively. Since the transformation $w(z)$ has a single pole in 0 (see (9)), the coefficient $n_{\mathrm{p}}$ is 0 . Therefore, the winding number $W\left(w(D), \lambda_{j}^{f}\right)$ will describe a number of unstable elements $n_{\mathrm{z}}$ of $w(z)-\lambda_{j}^{f}$. The contour $w(D)$ is based on (16) and is presented in Figure 2. Now:

$(\Rightarrow)$ Assume that the system is asymptotically stable. Then, all poles for all elements $w(z)-\lambda_{j}^{f}, j=1, \ldots, n$, are outside the interior domain $\underline{C}$ of the contour $D$. Therefore, $W\left(w(D), \lambda_{j}^{f}\right)=0 \forall j=1, \ldots, n$ and all $\lambda_{j}^{f}, j=1, \ldots, n$, are outside the area $w(D)$ bounded by $((1+a) /((1-a) h)$ $\left.\left(1-e^{-i \varphi}\right)\right)^{\alpha}$, which can be described by (10).

$(\Leftarrow)$ Assume that all eigenvalues $\lambda_{j}^{f}, j=1, \ldots, n$, are outside the instability area (10). Then, all eigenvalues are outside the contour $w(D)$ and the winding number $W\left(w(D), \lambda_{j}^{f}\right)=$ $0 \forall j=1, \ldots, n$. Therefore, the elements $w(z)-\lambda_{j}^{f}, j=1, \ldots, n$, do not generate poles in interior domain $\underline{C}$ of the contour $D$. So the system is asymptotically stable. This completes the proof.

A graphical presentation of the stability/instability areas is shown in Figure 3.
It can be seen from Figure 3 that the stability area depends on the weighting parameter $a$ of the Al-Alaoui equation. For $a=0$, we obtain the instability area as for the backward Euler operator (compare [13]) and increasing $a$ leads to enlargement of the instability area.

On the basis of Theorem 1, we can present a new analytical result as follows.

Theorem 2. The discrete-time fractional-order system (4) or (5) with $w(z)$ described by the Al-Alaoui operator (9), with $\alpha \in(0,2)$, is not asymptotically stable if and only if there exists any

$$
\begin{aligned}
\varphi_{j}^{f} & \in\left[-\alpha \frac{\pi}{2}, \alpha \frac{\pi}{2}\right] \wedge\left|\lambda_{j}^{f}\right| \\
& \leq\left(\frac{2(1+a)}{(1-a) h}\left|\cos \frac{\varphi_{j}^{f}}{\alpha}\right|\right)^{\alpha}, \quad j=1, \ldots, n,
\end{aligned}
$$

where $\left|\lambda_{j}^{f}\right|$ and $\varphi_{j}^{f}$ are the modulus and argument, respectively, of the $j$ - th $f$-pole of the system and $a \in[0,1)$ being the Al-Alaoui weighting coefficient.

Proof. Taking into account that the stability/instability bounding contour is given by (15), which can be presented in form of

$$
\begin{aligned}
w\left(e^{i \varphi}\right) & =\left(\frac{(1+a)}{(1-a) h} \sqrt{(1-\cos \varphi)^{2}+\sin ^{2} \varphi}\right)^{\alpha} e^{i \alpha \arctan \sin \varphi / 1-\cos \varphi} \\
& =\left(\frac{(1+a)}{(1-a) h} \sqrt{2(1-\cos \varphi)}\right)^{\alpha} e^{i \alpha \arctan (\tan \pi-\varphi / 2)} \\
& =\left(\frac{2(1+a)}{(1-a) h} \sin \frac{\varphi}{2}\right)^{\alpha} e^{i \alpha \pi-\varphi / 2},
\end{aligned}
$$

and introducing the resultant argument of $w\left(e^{i \varphi}\right)$, that is, $\varphi^{f}=\alpha(\pi-\varphi) / 2$, we obtain

$$
\begin{aligned}
w\left(e^{i \varphi^{f}}\right) & =\left(\frac{2(1+a)}{(1-a) h}\left|\sin \left(\frac{\pi}{2}-\frac{\varphi^{f}}{\alpha}\right)\right|\right)^{\alpha} e^{i \varphi^{f}} \\
& =\left(\frac{2(1+a)}{(1-a) h}\left|\cos \frac{\varphi^{f}}{\alpha}\right|\right)^{\alpha} e^{i \varphi^{f}} .
\end{aligned}
$$

Note that (21) presents the modulus of $w\left(e^{i \varphi^{f}}\right)$ as a function of its argument $\varphi^{f}$. Also note that for $\varphi \in[-\pi, \pi]$, the function $w\left(e^{i \varphi}\right)$ is redefined in $\varphi^{f} \in[-\alpha \pi / 2, \alpha \pi / 2]$. For the $f$-poles of the system with $\arg \left(\lambda_{j}^{f}\right) \notin[-\alpha \pi / 2, \alpha \pi / 2], j=$ $1, \ldots, n$, the $f$-poles are outside the instability area (see the stability/instability areas in Theorem 1) and the system is asymptotically stable.

$(\Rightarrow)$ Assume that the system is not asymptotically stable. Then, at least one $\lambda_{j}^{f}, j=1, \ldots, n$, lies inside or in the bound 


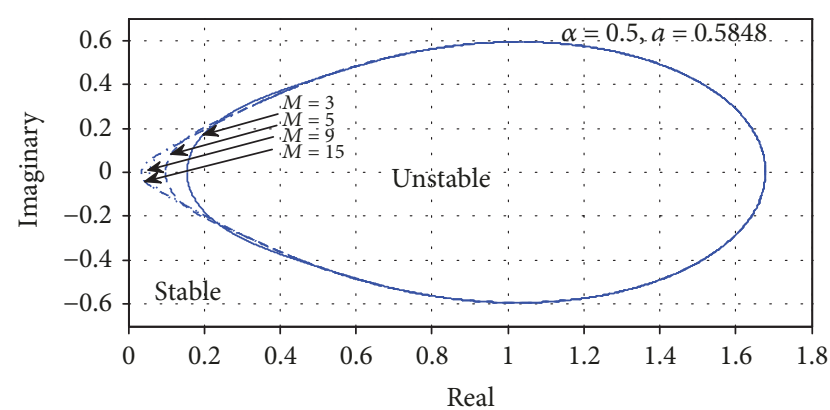

FIGURE 4: Stability/instability areas for $h=1$ and various $M$.

of the instability area described in Theorem 1 . Since the bound function $w\left(e^{i \varphi}\right)$ is redefined in $\varphi^{f} \in[-\alpha \pi / 2, \alpha \pi / 2]$, the argument of unstable eigenvalue $\lambda_{j}^{f}$ has to be $\varphi_{j}^{f} \in[-\alpha \pi /$ $2, \alpha \pi / 2]$. For $\arg \left(\lambda_{j}^{f}\right) \in[-\alpha \pi / 2, \alpha \pi / 2], j=1, \ldots, n$, the $f$ -poles are inside the instability area when the modulus of the $j$ th $f$-pole is lower (or equal) than the modulus of the stability/instability bounding contour $\left|w\left(e^{i \varphi^{f}}\right)\right|$ presented in (21) for the same angle $\varphi^{f}=\arg \left(\lambda_{j}^{f}\right)$. Therefore, there is at least one $f$-pole passing condition (18).

$(\Leftarrow)$ Assume that an $f$-pole $\lambda_{j}^{f}, j=1, \ldots, n$, of the system passes condition (18). Then, taking into account the stability/instability bound equation (21), the $f$-pole is inside (or in the bound) of the instability area. Taking into account Theorem 1, the system is not asymptotically stable. This completes the proof.

On the basis of Theorem 2, we can immediately present the following.

Theorem 3. The discrete-time fractional-order system (4) or (5) with $w(z)$ described by the Al-Alaoui operator (9), with $\alpha \in(0,2)$, is asymptotically stable if and only if

$$
\begin{aligned}
\varphi_{j}^{f} & \in\left(\alpha \frac{\pi}{2},-\alpha \frac{\pi}{2}\right) \vee\left|\lambda_{j}^{f}\right| \\
& >\left(\frac{2(1+a)}{(1-a) h}\left|\cos \frac{\varphi_{j}^{f}}{\alpha}\right|\right)^{\alpha}, \quad j=1, \ldots, n,
\end{aligned}
$$

where $\left|\lambda_{j}^{f}\right|$ and $\varphi_{j}^{f}$ are the modulus and argument, respectively, of the $j$-th $f$-pole of the system and $a \in[0,1)$ being the Al-Alaoui weighting coefficient.

Proof. Immediate from Theorem 2.

The results of Theorems 2 and 3 for the zero weighting coefficient of the Al-Alaoui operator $(a=0)$ specialize to those for the backward Euler-based discretization scheme presented in [13]. Also, on the basis of the results, we can easily present the stability criterion for the Tustin-based discretization scheme.
Theorem 4. The discrete-time fractional-order system (4) or (5) with $w(z)$ described by the Tustin operator (8), with $\alpha \in$ $(0,2)$, is asymptotically stable if and only if

$$
\varphi_{j}^{f} \in\left(\alpha \frac{\pi}{2},-\alpha \frac{\pi}{2}\right), \quad j=1, \ldots, n,
$$

where $\varphi_{j}^{f}$ is the argument of the $j$ - th $f$-pole of the system.

Proof. Consider the stability Theorem 3. Accounting that the Tustin-based approach is a special case of the AlAlaoui approach with $a \rightarrow 1_{-}$, we arrive at the following modulus condition:

$$
\left|\lambda_{j}^{f}\right|>\lim _{a \rightarrow 1_{-}}\left[\left(\frac{2(1+a)}{(1-a) h}\left|\cos \frac{\varphi_{j}^{f}}{\alpha}\right|\right)^{\alpha}\right], \quad j=1, \ldots, n,
$$

Taking into account that

$$
\lim _{a \rightarrow 1_{-}}\left[\left(\frac{2(1+a)}{(1-a) h}\left|\cos \frac{\varphi_{j}^{f}}{\alpha}\right|\right)^{\alpha}\right]=+\infty,
$$

then we have $\left|\lambda_{j}^{f}\right|<+\infty, j=1, \ldots, n$, and we arrive at condition (23).

Remark 1. Note that the results of Theorem 4 are the same as those for continuous-time systems [28, 29]. Thus, using the Tustin-based discretization scheme does not affect the stability conditions for discrete-time systems. However, the Tustin-based discretization is inferior with respect to approximation accuracy [10].

Remark 2. The results of Theorems 2 and 3 show that discretization of a stable continuous-time system using the Al-Alaoui-based approach guarantees the asymptotic stability of a discrete-time system. Moreover, for the unstable continuous-time system, we can select such a sampling period $h$ and weighting coefficient $a$ that lead to a stable discrete-time system.

\section{Finite-Length Implementation of Discretization Operators}

It is important that the "ideal" Al-Alaoui and Tustin operators are not applicable in practice due to infinite-length implementation of the discretization equations. Therefore, in practical applications, we approximate the two above operators by use of rational finite-length discrete-time transfer functions. Usually, the approximations are obtained through the continuous fraction expansion (CFE) method, but in the Tustin-approach, the Muir recursion can also be applied [10]. Regardless of the approximation method, the 
TABLE 1: Stability properties of discretized fractional-order system; Example 1.

\begin{tabular}{|c|c|c|c|c|c|}
\hline$j$ & $\varphi_{j}^{f}=\arg \left(\lambda_{j}^{f}\right)$ & $\left|\lambda_{j}^{f}\right|$ & $a$ & $\left(\frac{2(1+a)}{(1-a) h}\left|\cos \frac{\varphi_{j}^{f}}{\alpha}\right|\right)^{\alpha}$ & Comment \\
\hline 1 & $\pi$ & 1 & 0.5 & - & \\
\hline 2 & 0.67474 & 6.4031 & 0.5 & 5.1324 & Stable \\
\hline 3 & -0.67474 & 6.4031 & 0.5 & 5.1324 & \\
\hline 1 & $\pi$ & 1 & 0.6 & - & \\
\hline 2 & 0.67474 & 6.4031 & 0.6 & 5.9264 & Stable \\
\hline 3 & -0.67474 & 6.4031 & 0.6 & 5.9264 & \\
\hline 1 & $\pi$ & 1 & 0.7 & - & \\
\hline 2 & 0.67474 & 6.4031 & 0.7 & 7.0538 & Unstable \\
\hline 3 & -0.67474 & 6.4031 & 0.7 & 7.0538 & \\
\hline 1 & $\pi$ & 1 & 0.8 & - & \\
\hline 2 & 0.67474 & 6.4031 & 0.8 & 8.8896 & Unstable \\
\hline 3 & -0.67474 & 6.4031 & 0.8 & 8.8896 & \\
\hline 1 & $\pi$ & 1 & 1 & - & \\
\hline 2 & 0.67474 & 6.4031 & 1 & $\infty$ & Unstable \\
\hline 3 & -0.67474 & 6.4031 & 1 & $\infty$ & \\
\hline
\end{tabular}

rational discrete-time transfer function approximator $\widehat{w}(z)$ to $w(z)$ is obtained as follows:

$$
\widehat{w}(z)=\frac{\sum_{m=1}^{M} w_{m} z^{m}}{\sum_{m=1}^{M} v_{m} z^{m}} .
$$

In this case, the contour of the approximation $\widehat{w}(z)$ in the complex plane is (see $[2,24])$

$$
\widehat{w}\left(e^{i \varphi}\right)=\frac{\sum_{m=1}^{M} w_{m} e^{i m \varphi}}{\sum_{m=1}^{M} v_{m} e^{i m \varphi}} .
$$

Now, if curve (27) constitutes a simple closed curve in the complex plane, then the stability/instability areas with respect to $\lambda_{j}^{f}, j=1, \ldots, M$, are separated from each other by the contour. Exemplary stability/instability areas for $\alpha=0.5$, $h=1, a=0.71$, and various implementation lengths $M$ are presented in Figure 4.

\section{Simulation Examples}

Example 1. Consider a commensurate continuous-time fractional-order state space system as in (1) with

$$
\begin{aligned}
A_{f} & =\left[\begin{array}{ccc}
9 & -31 & -41 \\
1 & 0 & 0 \\
0 & 1 & 0
\end{array}\right], \\
B & =\left[\begin{array}{lll}
1 & 0 & 0
\end{array}\right]^{T}, \\
C & =\left[\begin{array}{lll}
0 & 0 & 1
\end{array}\right],
\end{aligned}
$$

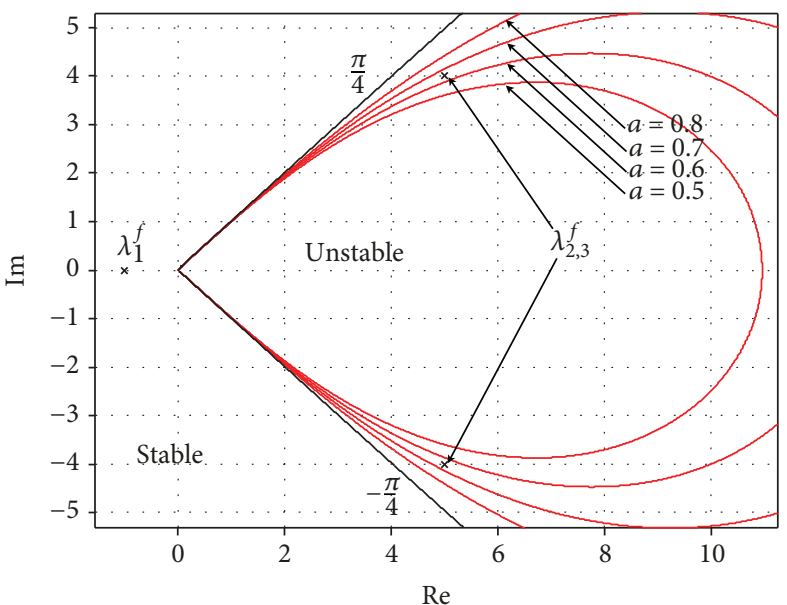

Figure 5: Stability/instability areas for various $a$; Example 1.

and $\alpha=0.5$. The system has three $f$-poles, i.e., $\lambda_{1}^{f}=-1$ and $\lambda_{2,3}^{f}=5 \pm i 4$. Note that since arg $\left(\lambda_{2,3}^{f}\right) \notin(\pi / 4,-\pi / 4)$, the system is unstable. The system is discretized by the use of the Al-Alaoui approach with the sampling period $h=0.05$ and four different values of the weighting coefficient $a=$ $0.5,0.6,0.7$, and 0.8 . The stability properties of the discretized system designed by use of Theorem 3 are presented in Table 1. Moreover, Table 1 shows the stability results for the Tustin method as a special case of the Al-Alaoui approach with $a=1$. The $f$-poles with the stability/instability areas are depicted in Figure 5.

It can be seen from Table 1 that even though the continuous-time system is unstable, the Al-Alaoui method with the weighting coefficients $a=0.5$ and $a=0.6$ can lead to the stable discrete-time system, but for the weighting coefficients $a=0.7$ and $a=0.8$, the discrete-time system 
TABLE 2: Stability properties of discretized fractional-order system; Example 2.

\begin{tabular}{cccccc}
\hline$j$ & $\varphi_{j}^{f}=\arg \left(\lambda_{j}^{f}\right)$ & $\left|\lambda_{j}^{f}\right|$ & $a$ & $\left(\frac{2(1+a)}{(1-a) h}\left|\cos \frac{\varphi_{j}^{f}}{\alpha}\right|\right)^{\alpha}$ & Comment \\
\hline 1 & 1.56236 & 0.55866 & 0.5 & 0.36025 & Stable \\
2 & -1.56236 & 0.55866 & 0.5 & 0.36025 & Unstable \\
\hline 1 & 1.56236 & 0.55866 & 0.8 & 1.87193 & 1.87193 \\
2 & -1.56236 & 0.55866 & 0.8 & & \\
\hline
\end{tabular}

still remains unstable. It is worth mentioning that the stability/instability of the discrete-time system is related to the unstable $f$-poles $\lambda_{2}^{f}$ and $\lambda_{3}^{f}$. Since the $f$-pole $\lambda_{1}^{f}$ is stable, the discrete-time counterpart will still be stable with respect to $\lambda_{1}^{f}$. The outcomes of Table 1 for the Tustin-based discretization scheme confirm the result of Theorem 4. Since the $f$-poles $\lambda_{1}^{f}$ and $\lambda_{2}^{f}$ are outside the instability areas for $a=$ 0.5 and $a=0.6$ and inside the instability areas for $a=0.7$ and $a=0.8$, the results of Figure 5 and Theorem 1 fully confirm the stability results specified in Table 1.

Example 2. Consider a commensurate continuous-time fractional-order state space system as in (1) with $\alpha=1.5$ and

$$
\begin{aligned}
A_{f} & =\left[\begin{array}{cc}
-0.78 & -0.3121 \\
1 & 0
\end{array}\right], \\
B & =\left[\begin{array}{ll}
1 & 0
\end{array}\right]^{T}, \\
C & =\left[\begin{array}{ll}
0 & 1
\end{array}\right],
\end{aligned}
$$

whose eigenvalues are $\lambda_{1,2}^{f}=-0.39 \pm 0.4 i$. Note that the system is unstable due to $\arg \left(\lambda_{1,2}^{f}\right) \notin(3 \pi / 4,-3 \pi / 4)$. The system is discretized by the use of the Al-Alaoui operator with the sampling period $h=1$ and two different values of the weighting coefficient $a=0.5$ and 0.8 . Stability results for the discretized system are presented in Table 2.

It can be seen from Table 2 that the discrete-time system is stable for $a=0.5$ but unstable for $a=0.8$.

Example 3. Consider fractional-order continuous-time system

$$
G(s)=\frac{1}{s^{0.9}-1.6 s^{0.6}-0.4611 s^{0.3}+2.1389},
$$

with $\alpha=0.3$ and three $f$-poles, i.e., $\lambda_{1}^{f}=-1$ and $\lambda_{2,3}^{f}=1.3 \pm i$ 0.67 . Note that $\arg \left(\lambda_{1,2,3}^{f}\right) \in(3 \pi / 20,-3 \pi / 20)$ and the system are stable. The system is discretized with the sampling period $h=0.05$ and the approximation of the Al-Alaoui operator as in (26), with $a=1 / 3$ and different implementation lengths $M=3$ and 5

$$
\widehat{w}_{3}(z)=\frac{2.6779 z^{3}-3.2135 z^{2}+0.57842 z+0.12711}{z^{3}-0.8 z^{2}-0.050667 z+0.04557},
$$

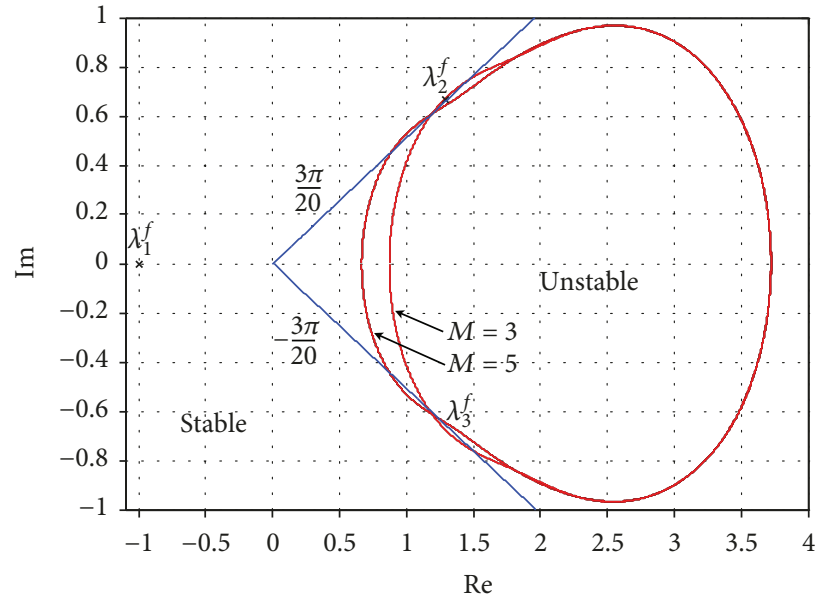

FIgURE 6: Stability/instability areas for different implementation lengths $M$; Example 3.

$$
\widehat{w}_{5}(z)=2.6779 z^{5}-4.9987 z^{4}+2.4147 z^{3}+0.1087 z^{2}-
$$

$0.18594 z+0.0041955 / z^{5}-1.4667 z^{4}+0.3684 z^{3}+0.17077 z^{2}$

$-0.037698 z-0.0034597$.

The stability areas and $f$-poles of the system are presented in Figure 6.

It can be seen from Figure 6 that although the original system is stable, the discretized one by use of finite implementation of the Al-Alaoui operator is unstable for $M=3$ and stable for $M=5$. Therefore, in contrast to the infinite-length Al-Alaoui operator, in some specific cases, the finite-length implementation of the Al-Alaoui discretizer for a stable continuous time system can lead to an unstable discrete-time system.

All the results presented in the above examples have been confirmed by a variety of BIBO stability experiments.

Remark 3. Matlab-scripted files for the above examples are available from the web: doi:10.5281/zenodo.1414176.

\section{Conclusion}

This paper has offered new, simple, graphical, and analytical stability/instability conditions for continuous-time commensurate fractional-order systems discretized by the use of the Al-Alaoui and Tustin operators. Firstly, theoretical stability condition for the Al-Alaoui operator has been given in a graphical way, which is then used in simple, analytical stability tests for both Al-Alaoui and Tustin approaches. Finally, 
the stability of discrete-time systems by use of finite-length approximations of the Al-Alaoui and Tustin discretizers has been discussed. Simulation examples fully confirm the original stability results obtained.

It is important that the fractional-order stability analysis presented in the paper is based on the eigenvalues of the state matrix, exactly as for the integer-order systems. This method is very useful; however, in case of high dimensions of the state matrices, calculation of eigenvalues may lead to numerical problems.

\section{Conflicts of Interest}

The authors declare that they have no conflicts of interest.

\section{References}

[1] I. Petráš, Fractional-Order Nonlinear Systems: Modeling, Analysis and Simulation, Nonlinear Physical Science, Springer, Berlin, Heidelberg, 2011.

[2] M. Siami, M. S. Tavazoei, and M. Haeri, "Stability preservation analysis in direct discretization of fractional order transfer functions," Signal Processing, vol. 91, no. 3, pp. 508-512, 2011.

[3] Y. Q. Chen and K. L. Moore, "Discretization schemes for fractional-order differentiators and integrators," IEEE Transactions on Circuits and Systems I: Fundamental Theory and Applications, vol. 49, no. 3, pp. 363-367, 2002.

[4] Y. Q. Chen and B. M. Vinagre, "A new IIR-type digital fractional order differentiator," Signal Processing, vol. 83, no. 11, pp. 2359-2365, 2003.

[5] R. Stanisławski, M. Rydel, and M. Gałek, "Implementation issues in discretization of fractional-order derivative using the Al-Alaoui operator," in 2016 21st International Conference on Methods and Models in Automation and Robotics (MMAR), pp. 844-847, Miedzyzdroje, Poland, 2016.

[6] B. M. Vinagre, Y. Q. Chen, and I. Petráš, "Two direct Tustin discretization methods for fractional-order differentiator/integrator," Journal of the Franklin Institute, vol. 340, no. 5, pp. 349-362, 2003.

[7] P. Bania and J. Baranowski, "Laguerre polynomial approximation of fractional order linear systems," in Advances in the Theory and Applications of Non-integer Order Systems, vol. 257 of Lecture Notes in Electrical Engineering, pp. 171-182, Springer, Heidelberg, Germany, 2013.

[8] J. Baranowski, W. Bauer, M. Zagórowska, and P. Piatek, "On digital realizations of non-integer order filters," Circuits, Systems, and Signal Processing, vol. 35, no. 6, pp. 2083-2107, 2016.

[9] M. Bishehniasar, S. Salahshour, A. Ahmadian, F. Ismail, and D. Baleanu, "An accurate approximate-analytical technique for solving time-fractional partial differential equations," Complexity, vol. 2017, Article ID 8718209, 12 pages, 2017.

[10] R. Stanisławski, K. J. Latawiec, and M. Łukaniszyn, “A comparative analysis of Laguerre-based approximators to the Grünwald-Letnikov fractional-order difference," Mathematical Problems in Engineering, vol. 2015, Article ID 512104, 10 pages, 2015.

[11] A. Dzieliński and D. Sierociuk, "Stability of discrete fractional order state-space systems," Journal of Vibration and Control, vol. 14, no. 9-10, pp. 1543-1556, 2008.
[12] S. Guermah, S. Djennoune, and M. Bettayeb, "A new approach for stability analysis of linear discrete-time fractional-order systems," in New Trends in Nanotechnology and Fractional Calculus Applications, D. Baleanu, Z. B. Guvenc, and J. A. T. Machado, Eds., pp. 151-162, Springer, Dordrecht, Netherlands, 2010.

[13] R. Stanisławski, "New results in stability analysis for LTI SISO systems modeled by GL-discretized fractional-order transfer functions," Fractional Calculus and Applied Analysis, vol. 20, no. 1, 2017.

[14] S. B. Stojanović and D. L. Debeljković, "Simple stability conditions of linear discrete time systems with multiple delay," Serbian Journal of Electrical Engineering, vol. 7, no. 1, pp. 6979, 2010.

[15] R. Stanisławski and K. J. Latawiec, "Stability analysis for discrete-time fractional-order LTI state-space systems. Part I: new necessary and sufficient conditions for the asymptotic stability," Bulletin of the Polish Academy of Sciences Technical Sciences, vol. 61, no. 2, pp. 353-361, 2013.

[16] R. Stanisławski and K. J. Latawiec, "Stability analysis for discrete-time fractional-order LTI state-space systems. Part II: new stability criterion for FD-based systems," Bulletin of the Polish Academy of Sciences Technical Sciences, vol. 61, no. 2, pp. 363-370, 2013.

[17] M. Busłowicz, "Robust stability of positive discrete-time linear systems of fractional order," Bulletin of the Polish Academy of Sciences Technical Sciences, vol. 58, no. 4, pp. 567-572, 2010.

[18] M. Busłowicz and T. Kaczorek, "Simple conditions for practical stability of positive fractional discrete-time linear systems," International Journal of Applied Mathematics and Computer Science, vol. 19, no. 2, pp. 263-269, 2009.

[19] T. Kaczorek, "New stability tests of positive standard and fractional linear systems," Circuits and Systems, vol. 2, no. 4, pp. 261-268, 2011.

[20] T. Kaczorek, Selected Problems of Fractional Systems Theory, Springer, Berlin, Heidelberg, 2011.

[21] D. Mozyrska and M. Wyrwas, "The $\mathscr{Z}$-transform method and delta type fractional difference operators," Discrete Dynamics in Nature and Society, vol. 2015, Article ID 852734, 12 pages, 2015.

[22] P. Ostalczyk, "Equivalent descriptions of a discrete-time fractional-order linear system and its stability domains," International Journal of Applied Mathematics and Computer Science, vol. 22, no. 3, pp. 533-538, 2012.

[23] P. Ostalczyk, Discrete Fractional Calculus: Applications in Control and Image Processing, World Scientific, 2016.

[24] K. Oprzedkiewicz, R. Stanisławski, E. Gawin, and W. Mitkowski, "A new algorithm for a CFE-approximated solution of a discrete-time noninteger-order state equation," Bulletin of the Polish Academy of Sciences Technical Sciences, vol. 65, no. 4, pp. 429-437, 2017.

[25] M. A. Al-Alaoui, "Novel digital integrator and differentiator," Electronics Letters, vol. 29, no. 4, pp. 376-378, 1993.

[26] M. A. Al-Alaoui, "Al-Alaoui operator and the new transformation polynomials for discretization of analogue systems," Electrical Engineering, vol. 90, no. 6, pp. 455-467, 2008.

[27] R. Lopez, Advanced Engineering Mathematics, Addison Wesley Publishing Company, Boston, MA, USA, Second edition, 2001. 
[28] D. Matignon, "Stability results for fractional differential equations with applications to control processing," in Computational Engineering in Systems and Applications Multiconference, pp. 963-968, Lille, France, 1996.

[29] D. Matignon, "Stability properties for generalized fractional differential systems," ESAIM: Proceedings, vol. 5, pp. 145$158,1998$. 


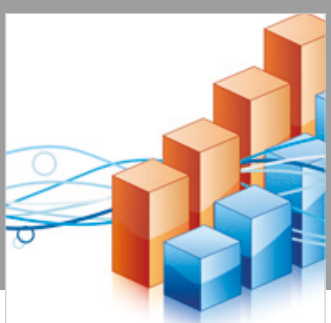

Advances in

Operations Research

\section{-n-m}
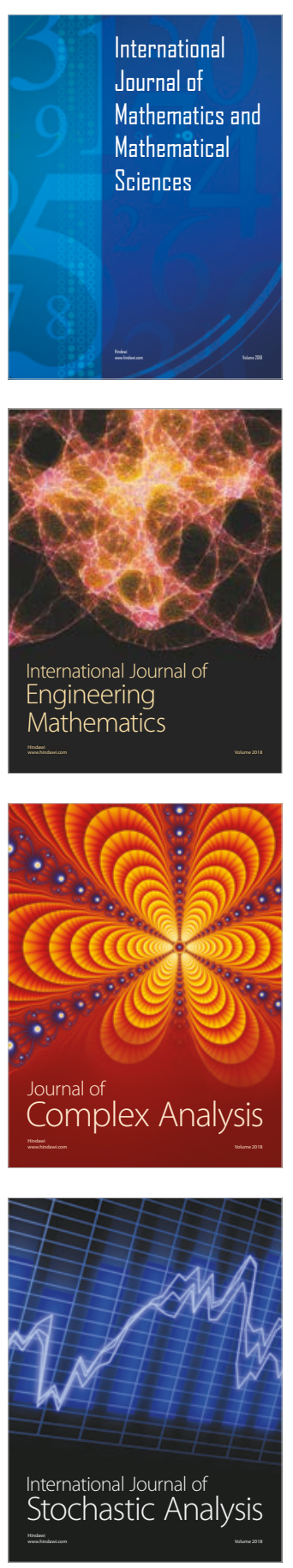
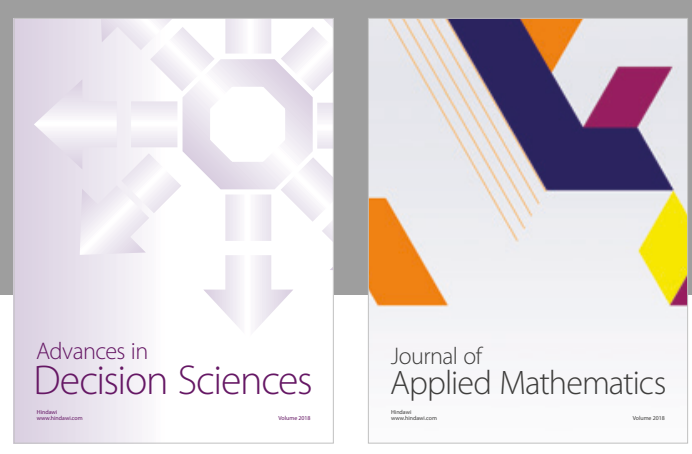

Journal of

Applied Mathematics
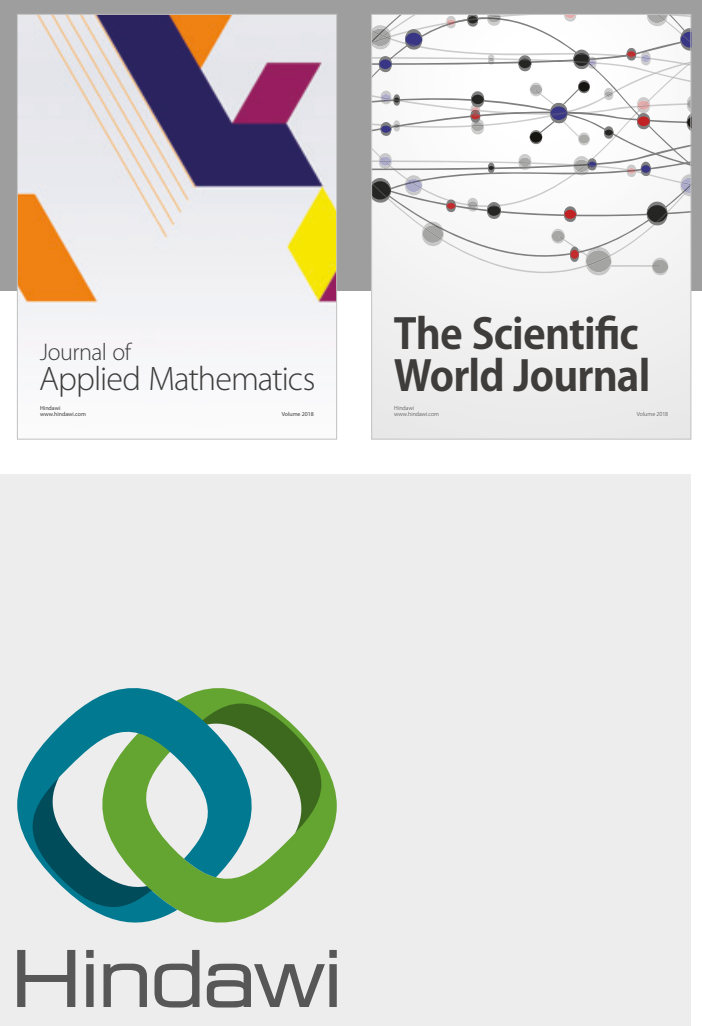

Submit your manuscripts at

www.hindawi.com

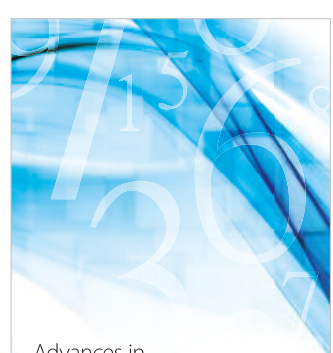

Advances in
Numerical Analysis
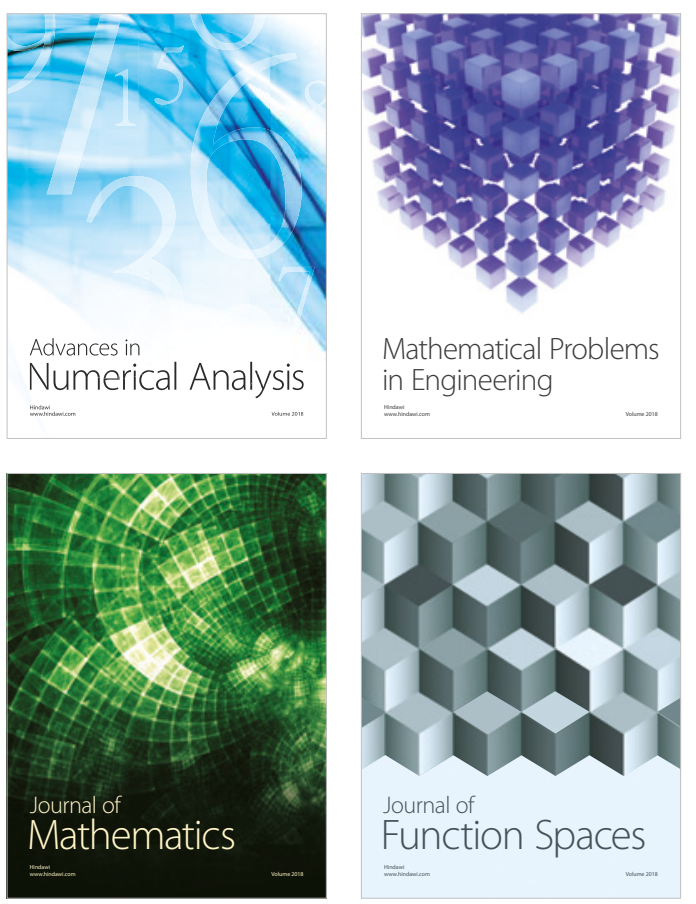

Mathematical Problems in Engineering

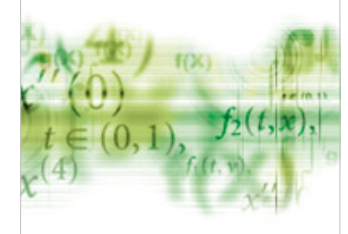

International Journal of

Differential Equations

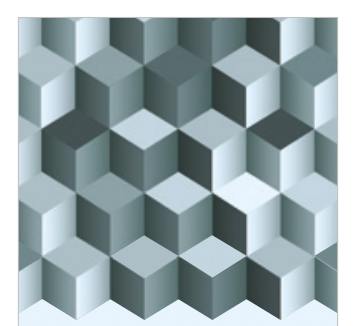

Journal of

Function Spaces
The Scientific

World Journal

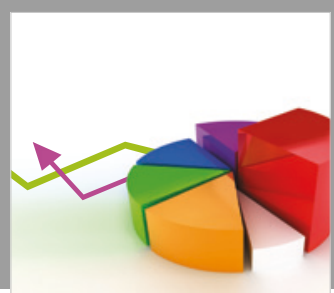

Journal of

Probability and Statistics
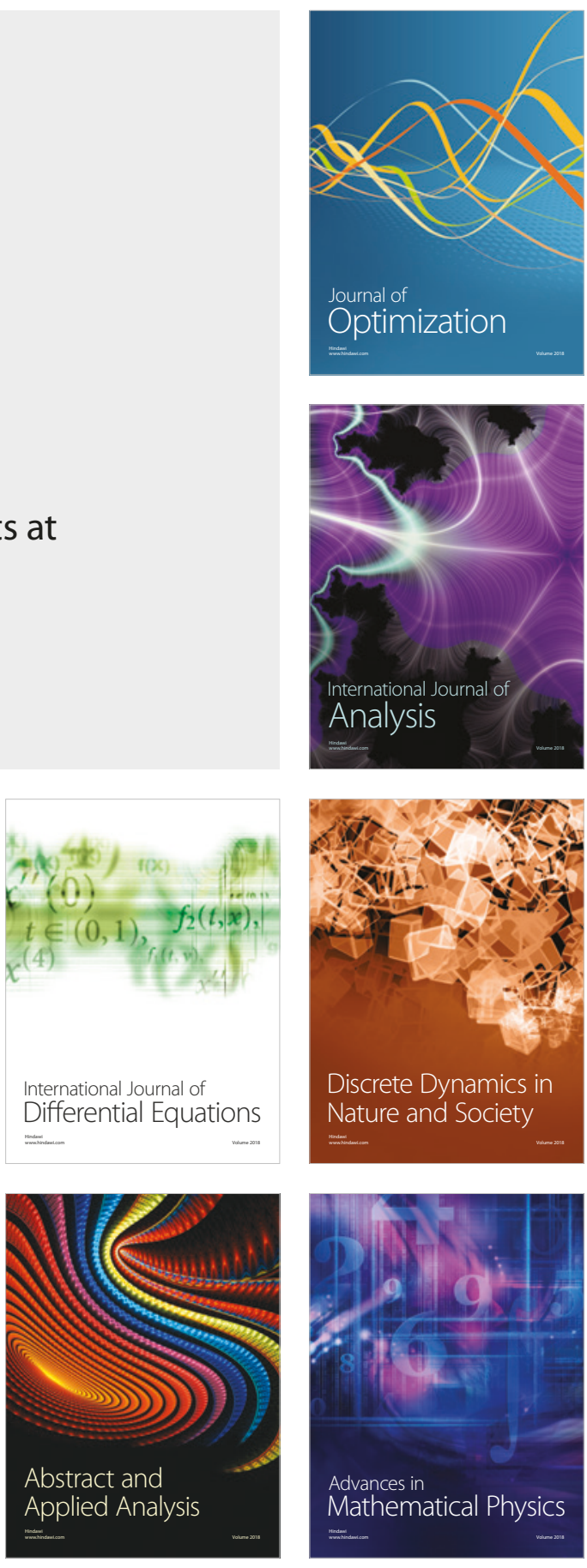